\title{
Negative Entropy of Mixing for Vanadium-Platinum Solutions
}

\author{
O. Delaire, T. Swan-Wood, and B. Fultz \\ California Institute of Technology, W. M. Keck Laboratory, mail 138-78, Pasadena, California 91125, USA
}

(Received 9 April 2004; published 29 October 2004)

\begin{abstract}
The phonon densities of states for pure vanadium and the solid solutions $\mathrm{V}-6.25 \% \mathrm{Ni}, \mathrm{Pd}, \mathrm{Pt}$ were determined from inelastic neutron scattering measurements. The solute atoms caused a large stiffening of the phonons, resulting in large, negative vibrational entropies of mixing. For $\mathrm{V}-6.25 \% \mathrm{Pt}$, the negative vibrational entropy of mixing exceeds the conventional positive chemical entropy of mixing. This negative total entropy of mixing should extend to lower concentrations of $\mathrm{Pt}$, and the effect on the bcc solvus line is discussed. The experimental data were inverted to obtain interatomic force constants by using a Born-von Kármán model with an iterative optimization algorithm. The stiffening of bonds responsible for the decrease of entropy was found to occur mainly in first-nearest-neighbor solute-host bonds, and correlates in part with the solute metallic radius.
\end{abstract}

DOI: 10.1103/PhysRevLett.93.185704

Mixing creates structural disorder, so the entropy of a mixed system is expected to be larger than an unmixed system. The well-known configurational entropy of mixing in the point approximation

$$
S_{\mathrm{cf}}^{\operatorname{mix}}=-k_{\mathrm{B}}[c \ln c+(1-c) \ln (1-c)]
$$

is always positive ( $c$ is concentration, $0<c<1$ ). Its contribution to the free energy $F=E-T S$ therefore promotes solubility at higher temperatures, and, in equilibrium with a second phase of lower entropy, the solubility limit of a solid solution should increase with temperature. This is typically observed, but exceptions are noteworthy, including unexplained cases where solid solubility decreases over a range of temperature, termed "retrograde" solubility.

Experimental and theoretical investigations have shown that differences in vibrational entropy play an important role in the relative thermodynamic stabilities of solid phases [1-5]. There is now a widespread interest in better understanding the effects of vibrational entropy on phase diagrams, an active topic of $a b$ initio investigations. Here we show, to our knowledge for the first time, a negative entropy of mixing at rather low solute concentrations, caused by the entropy of atomic vibrations. The thermal stability of structurally ordered states and disordered states is thereby reversed.

Although the solubility of $\mathrm{Ni}$ in the body-centered cubic (bcc) V-rich phase is qualitatively consistent with the entropy of Eq. (1), the elements below $\mathrm{Ni}$ in the periodic table, Pd and especially Pt, have similar phase diagrams but show a much weaker temperature dependence of solubility in bcc V [6]. Owing to its incoherent neutron cross section and cubic crystal structure, vanadium is the ideal element for measuring a phonon densityof-states (DOS) by inelastic neutron scattering [7], as are V-rich bcc solid solutions. From the phonon DOS the vibrational entropy and other phonon thermodynamic functions can be obtained, at least at low temperatures. Previous studies of resonance modes of heavy solutes in
PACS numbers: $64.75 .+\mathrm{g}, 61.12 .-\mathrm{q}, 63.70 .+\mathrm{h}$

vanadium suggest important effects on phonon thermodynamics [8-11].

Alloys were prepared by arc melting under a highpurity argon atmosphere. The ingots of V-Ni, V-Pd, and V-Pt were subsequently cold rolled to the desired thicknesses and annealed in vacuum at $950{ }^{\circ} \mathrm{C}$ for $1 \mathrm{~h}$ and air cooled in their quartz ampoules. X-ray diffractometry showed the samples to be bcc solid solutions.

The longitudinal $\left(c_{L}\right)$ and transverse $\left(c_{T}\right)$ sound velocities were measured by transit times of ultrasonic pulses. The shear and bulk moduli $G=\rho c_{T}^{2}$ and $B=\rho c_{L}^{2}-\frac{4}{3} G$ were obtained from the acoustic velocities and the alloy densities. The results in Table I show only a slight stiffening in bulk modulus in the alloys V-Pt and V-Ni compared with pure V, and no measurable effect in V-Pd. On the other hand, the solutes cause a substantial stiffening of $G$. Compared to pure V, the relative stiffenings of $G$ for $\{\mathrm{V}-$ $\mathrm{Ni}, \mathrm{V}-\mathrm{Pd}, \mathrm{V}-\mathrm{Pt}\}$ are, sequentially, $\{6 \%, 16 \%, 30 \%\}$.

Inelastic neutron scattering spectra were measured using the IPNS-LRMECS time-of-flight chopper spectrometer at the Argonne National Laboratory. The incident energy was $55 \mathrm{meV}$ and the detector coverage allowed for momentum transfers between 0.5 and $9 \AA^{-1}$ at zero energy transfer. The FWHM energy resolution was $1.1 \mathrm{meV}$ at $40 \mathrm{meV}$ positive energy transfer, increasing to

TABLE I. Measured and calculated elastic moduli.

\begin{tabular}{lcccc}
\hline \hline & $\begin{array}{c}B \text { meas. } \\
(\mathrm{GPa})\end{array}$ & $\begin{array}{c}B \text { calc. } \\
(\mathrm{GPa})\end{array}$ & $\begin{array}{c}G \text { meas. } \\
(\mathrm{GPa})\end{array}$ & $\begin{array}{c}G \text { calc. } \\
(\mathrm{GPa})\end{array}$ \\
\hline $\mathrm{V}$ & $149 \pm 7$ & 163 & $46 \pm 0.7$ & 35.2 \\
$\mathrm{~V}^{\mathrm{a}}$ & 155.2 & & 42.55 & \\
$\mathrm{~V}^{\mathrm{b}}$ & 157.12 & & 43.768 & \\
$\mathrm{~V}-\mathrm{Ni}$ & $163 \pm 8$ & 174 & $48.6 \pm 0.9$ & 40.8 \\
$\mathrm{~V}-\mathrm{Pd}$ & $150 \pm 6$ & 159 & $53.4 \pm 0.9$ & 40.9 \\
$\mathrm{~V}-\mathrm{Pt}$ & $167 \pm 7$ & 170 & $59 \pm 1$ & 47.1 \\
\hline \hline
\end{tabular}

${ }^{\text {a }}$ Reference [12].

${ }^{\mathrm{b}}$ Reference [13]. 
$2.8 \mathrm{meV}$ at the elastic line. All spectra were normalized by the total incident flux and sample mass, and were corrected for detector efficiency and time-independent background. The spectrum from the empty sample container was subtracted, and the elastic peak was removed. A standard procedure was used to remove effects of temperature and multiphonon scattering [14]. This analysis uses the incoherent-scattering approximation, which should be well satisfied for solid solutions of vanadium when solutes that scatter coherently are distributed randomly. For pure elements of cubic crystals, this analysis provides the phonon DOS [7].

Because different elements and isotopes have different neutron scattering cross sections, the phonon DOS curves obtained from this data reduction procedure are "neutron weighted"- phonon modes involving large displacements of elements with larger cross sections tend to be overemphasized. We call such a spectrum a generalized phonon DOS (gDOS). The intensity of phonon scattering is proportional to the ratio of the elemental cross section divided by mass, and the ratios for our solute atoms with respect to vanadium are $\{3.1,0.4,0.6\}$ for $\{\mathrm{Ni}, \mathrm{Pd}, \mathrm{Pt}\}$ [15]. For small solute concentrations, neutron weighting has only small effects on the phonon DOS. Nevertheless, a correction for this effect is possible when the full lattice dynamics can be determined, and the full lattice dynamics also permits assessments of partial DOS curves as explained below.

A Born-von Kármán model of lattice dynamics $[16,17]$ was developed for a 16 -atom cubic supercell containing $8 \mathrm{bcc}$ unit cells of all $\mathrm{V}$ atoms, except the central atom was a solute atom $X(X=\mathrm{Ni}, \mathrm{Pd}$, or Pt) (see Fig. 1). Three sets of independent force constants were included in the model, corresponding to forces between the solute and vanadium atoms, force constants between the vanadium atoms that were first-nearest neighbors (1NN) of the solute atom, and force constants between matrix vanadium atoms. All three sets of force constants included pairs up to $5 \mathrm{NN}$. The supercell dynamics allowed calculation of both the true phonon DOS and the neutronweighted phonon gDOS by finding a best fit to the experimental gDOS. Six tensorial interatomic force constants $\Phi_{\alpha, \beta}^{\kappa, \kappa^{\prime}}$, corresponding to the three arrows in Fig. 1, were iteratively optimized using Powell's algorithm [18]. (The $\Phi_{\alpha, \beta}^{\kappa, \kappa^{\prime}}$ for more distant neighbors were fixed at values obtained from bcc vanadium.) The gDOS histograms were calculated for $1 \times 10^{3}$ random $\vec{q}$ points (as suggested by a convergence study), and broadened by an instrument resolution function. The optimization criterion for minimizing the deviation between the calculated and experimental gDOS curves used a $\chi^{2}$ that accounted for the errors from experimental counting statistics. (Some details of the inversion procedure were given previously [19].) The DOS of pure vanadium was also inverted and yielded a force-constant tensor in very good agreement with prior results up to $4 \mathrm{NN}$ pairs [20].

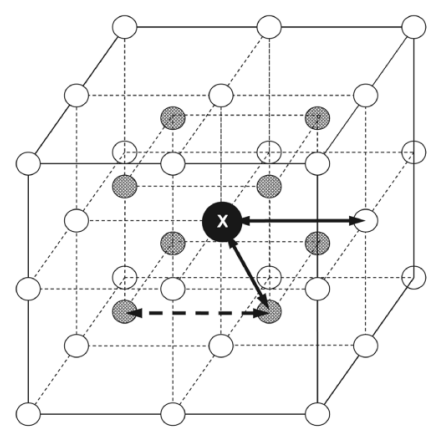

FIG. 1. Supercell used in lattice dynamics inversion. The central black circle is the solute atom $X$. Small dark atoms are $1 \mathrm{NN}$ vanadium atoms. Arrows indicate force constants optimized in the simulation. Solid arrows: $X-\mathrm{V}$ force constants. Dashed arrow: V1-V1 force constants.

A test of these optimized $\Phi_{\alpha, \beta}^{\kappa, \kappa^{\prime}}$ is to calculate the elastic constants $c_{11}, c_{12}$, and $c_{44}$ and the elastic moduli $B=\left(c_{11}+2 c_{12}\right) / 3$ and $G=c_{44}$, using the Born-Huang relations [16], which were evaluated up to $3 \mathrm{NN}$ pairs for a bcc lattice. Supercell alloy averages for $B$ and $G$ were obtained by averaging the fraction of $X-\mathrm{V}$ or $\mathrm{V}-\mathrm{V}$ pairs in each shell. Values of bulk and shear modulus obtained from the inverted force constants are compared with our results from ultrasonic measurements in Table I. The changes of elastic moduli with respect to pure vanadium are well reproduced.

The ratio of the calculated gDOS and DOS curves is the neutron-weight distribution that was used to obtain the corrected experimental DOS curves in Fig. 2. A large stiffening is observed in the DOS upon alloying, with a positive shift in the cutoff energy in excess of $3 \mathrm{meV}$ for $\mathrm{Pt}$ solutes. This effect is stronger down the column of the periodic table from $\mathrm{Ni}$ to $\mathrm{Pd}$ to $\mathrm{Pt}$, indicating a correlation with the size and/or mass of the solute species. Calculated DOS curves resulting from our inversion procedure are also presented in Fig. 2.

Partial DOS curves calculated with the optimized force constants are shown in Fig. 3. A striking feature is the low-energy resonance mode observed in the $\mathrm{Pt}$ and Pd partial DOS curves around $12 \mathrm{meV}$. Resonance modes can occur when the solute mass is significantly larger than that of the host atoms [17,21]. In both V-Pt and V$\mathrm{Pd}$, an extra solute peak is also observed at high energies, somewhat above the cutoff energy of pure vanadium, whereas few modes are present at intermediate energies. For both V-Pd and V-Pt, the $3 \mathrm{NN}$ vanadium partial DOS is similar to the DOS of pure vanadium, but the $1 \mathrm{NN}$ partial DOS shows a large stiffening. With eight such $1 \mathrm{NN}$ vanadium atoms about each solute atom, this stiffening alters significantly the total phonon DOS.

From the optimized tensorial force constants, we calculated the longitudinal and transverse components associated with a central potential [17]. A prominent trend was a very large change in the 1NN solute-host forces, 


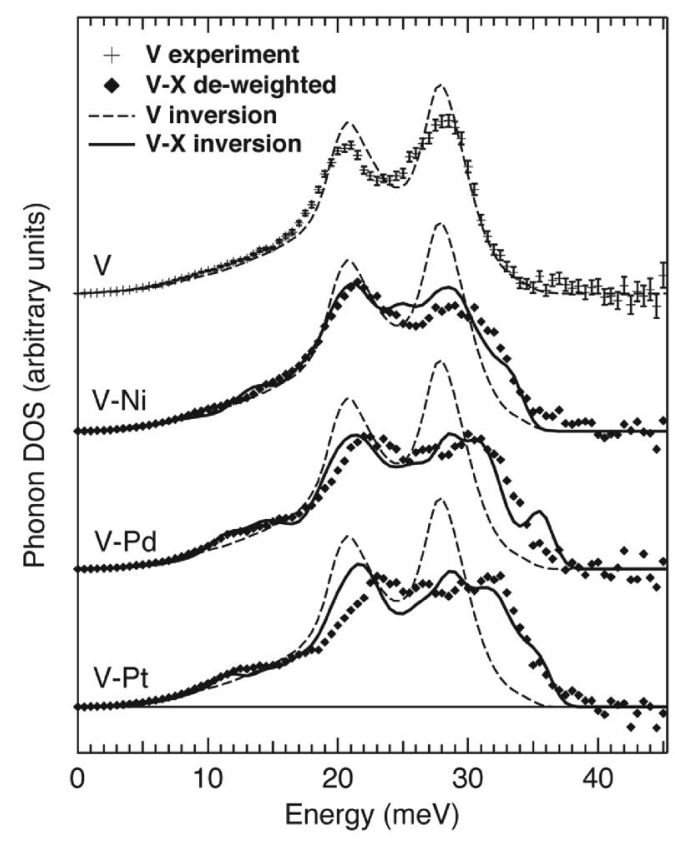

FIG. 2. Points: experimental DOS curves for pure vanadium and alloys, corrected for neutron weighting. Dashed line: V DOS from minimization algorithm. Solid line: calculated DOS curve from the dynamics model.

notably the longitudinal stiffness, $L 1$, of $1 \mathrm{NN} X$-V pairs. This is correlated to the solute metallic radius in Fig. 4 (the bcc metallic radii of $\mathrm{Ni}, \mathrm{V}, \mathrm{Pd}$, and $\mathrm{Pt}$ are 121.3, 131.0, 133.9 , and $135.0 \mathrm{pm}$, respectively [22]). This force constant is much larger in V-Pt and V-Pd than in pure $\mathrm{V}$, while it is much smaller in V-Ni. This result indicates that $L 1$ increases or decreases dramatically when the central $\mathrm{V}$ atom is replaced by a larger $(\mathrm{Pd}, \mathrm{Pt})$ or smaller $(\mathrm{Ni})$ solute

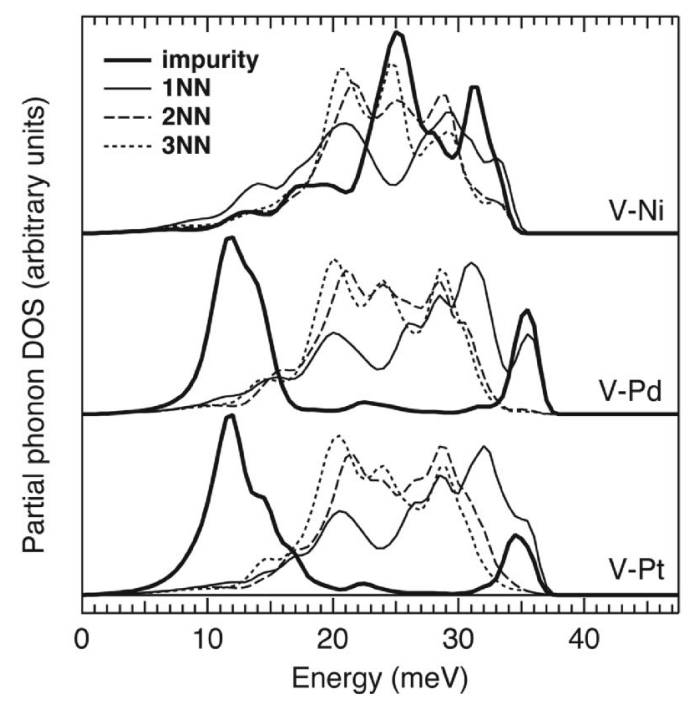

FIG. 3. Partial DOS curves for the solute atom and its V neighbors up to $3 \mathrm{NN}$ shell, obtained from the optimized supercell lattice dynamics. atom. A similar trend was observed for $L 2$. Conversely, the transverse stiffness, $T 1$, clearly follows an opposite trend, decreasing as the size of the solute atom gets larger. The trend in $L 1$ is expected because a large substitutional solute atom compresses the bonds to neighboring vanadium atoms, increasing the longitudinal bond stiffness. The opposite effect in $T 1$ can be understood because the compressed rows of atoms passing through the impurity atom are unstable against lateral displacements of atoms in the row, as was reported for interstitial defects [23]. The changes in $L 1$ and $T 1$ correlate well with the difference in metallic radius between the host and solute atom, as seen in Fig. 4. The relaxation of atomic positions around the solute atom is not taken into account in our lattice dynamics model, however. The distortions in bond length extend beyond the 1NN shell of the solute, so our calculated changes in the bond stiffnesses account for cumulative effects over longer physical distances.

The vibrational entropy $S_{\text {vib }}$, in the quasiharmonic approximation is

$S_{\text {vib }}=3 k_{\mathrm{B}} \int_{0}^{E_{\max }}\left[\left(n_{E}+1\right) \ln \left(n_{E}+1\right)-n_{E} \ln \left(n_{E}\right)\right] \times g(E) d E$,

where $n_{E}$ is the Bose-Einstein distribution and $g(E)$ is the DOS, both for the same temperature. For each alloy, the DOS used in Eq. (2) was the experimental DOS curve corrected for neutron weighting as discussed above. The vibrational entropy of mixing, $S_{\mathrm{vib}}^{\mathrm{mix}}(c)$, was calculated as the difference in vibrational entropy between alloys and the pure elements of appropriate proportions, using some pure element phonon data from the literature [24-26]. Results for room temperature are listed in Table II. At higher temperatures $S_{\mathrm{vib}}^{\mathrm{mix}}$ should be even more negative owing to the behavior of $n_{E}(T)$. The error was obtained from the experimental error of our measured DOS curves.

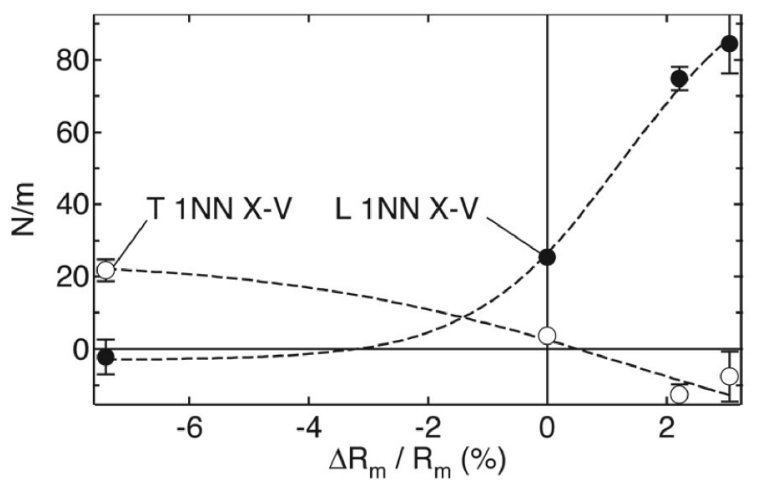

FIG. 4. Optimized longitudinal (L) and transverse (T) 1NN solute-vanadium force constants. The $x$ axis is the relative difference in metallic radius between solute species and vanadium, representing (left to right) $\mathrm{Ni}, \mathrm{V}, \mathrm{Pd}$, and $\mathrm{Pt}$. Lines are drawn to guide the eye. 
TABLE II. Vibrational entropy of mixing from the corrected DOS curves of the alloy and DOS curves of pure elements, evaluated at $295 \mathrm{~K}$.

\begin{tabular}{lcc}
\hline \hline Sample & $E_{\max }(\mathrm{meV})$ & $S_{\mathrm{vib}}^{\operatorname{mix}}\left(k_{\mathrm{B}} /\right.$ atom $)$ \\
\hline $\mathrm{V}-6.25 \% \mathrm{Ni}$ & 36 & $-0.082 \pm 0.005$ \\
$\mathrm{~V}-6.25 \% \mathrm{Pd}$ & 38 & $-0.185 \pm 0.005$ \\
$\mathrm{~V}-6.25 \% \mathrm{Pt}$ & 40 & $-0.272 \pm 0.005$ \\
\hline \hline
\end{tabular}

For dissolving 6.25 at. \% $\mathrm{Pd}$ or $\mathrm{Pt}$ in $\mathrm{V}$, the large negative vibrational entropy of mixing has unexpected thermodynamic consequences. For V-Pt the value of $S_{\text {vib }}^{\text {mix }}(0.0625)=-0.272 k_{\mathrm{B}} /$ atom exceeds the configurational entropy of mixing $S_{\mathrm{cf}}^{\mathrm{mix}}(0.0625)=+0.234 k_{\mathrm{B}} /$ atom of Eq. (1). For a V-6.25 at.\% Pt alloy, the total entropy of mixing is therefore negative. Assuming the effects of solute atoms on the phonon spectrum are linear in the solute concentration at these low concentrations, the entropy of mixing will be negative for all concentrations exceeding 3.4 at. \% Pt or 13 at.\% Pd. The configurational entropy of mixing will dominate at lower concentrations, owing to its logarithmic singularity. Nevertheless, the first term in Eq. (1) is largest at low concentrations, so the composition where $S_{\text {total }}^{\text {mix }}=0$ decreases approximately exponentially with the vibrational entropy contribution of the solute atom (assuming $S_{\text {vib }}$ is linear in concentration). A hypothetical solute causing twice the effect of $\mathrm{Pt}$ would have a negative entropy of mixing for compositions as low as 0.04 at. \%. In this case the increase of $S_{\text {vib }}^{\text {mix }}$ with temperature [through $n_{E}(T)$ of Eq. (2)] could lead to retrograde solubility, and there were hints that a negative vibrational entropy may contribute to retrograde solubility [27].

In summary, the stiffening of the Pt-V $1 \mathrm{NN}$ longitudinal force constant is responsible for a large negative vibrational entropy of mixing of Pt in V. For Pt concentrations of only a few atomic percent, the total entropy of mixing is therefore negative. This negative entropy of mixing affects the solubility of $\mathrm{Pt}$ in V. The bcc solvus curve has a much stronger temperature dependence for $\mathrm{V}$ $\mathrm{Ni}$ than for V-Pd or V-Pt, as expected from the present trends of the vibrational entropies of mixing in these three materials (for V-Pt the bcc solvus has little temperature dependence over $2000 \mathrm{~K}$ [6]). The larger negative vibrational entropy of mixing of Pt compared to Pd may also contribute to the lower solubility of Pt than Pd. An overall negative entropy of mixing may exist for other solid solutions of large solute atoms, even at low solute concentrations. A negative entropy of mixing for the solid solution gives thermal stability to ordered states, suppressing the formation of structurally disordered states at elevated temperatures.

We thank J. G-W. Lin for help with software development. This work was supported by the Department of
Energy through the Basic Energy Sciences Grant No. DEFG03-0346055 and BES-MS, W-31-109-ENG-38.

[1] L. Anthony, J. K. Okamoto, and B. Fultz, Phys. Rev. Lett. 70, 1128 (1993).

[2] B. Fultz et al., Phys. Rev. B 52, 3315 (1995).

[3] V. Ozolins, C. Wolverton, and A. Zunger, Phys. Rev. B 57, 6427 (1998); 58, 5897 (1998).

[4] A. van de Walle and G. Ceder, Rev. Mod. Phys. 74, 11 (2002).

[5] Z. Gurskii and J. Krawczyk, Physica (Amsterdam) 304B, 319 (2001).

[6] Phase Diagrams for Binary Alloys, edited by $\mathrm{H}$. Okamoto (ASM International, Materials Park, $\mathrm{OH}$, 2000).

[7] G. Placzek and L. Van Hove, Phys. Rev. 93, 1207 (1954).

[8] B. Mozer, K. Otnes, and C. Thaper, Phys. Rev. 152, 535 (1966).

[9] G. F. Syrykh, G. M. Zemlyanov, N. A. Chernoplekov, and V. M. Koltygin, Zh. Eksp. Teor. Fiz. 73, 313 (1977) [Sov. Phys. JETP 46, 162 (1977)].

[10] G. F. Syrykh et al., Zh. Eksp. Teor. Fiz. 70, 353 (1976) [Sov. Phys. JETP 43, 183 (1976)].

[11] G. F. Syrykh et al., Fiz. Tverd. Tela (Leningrad) 26, 3139 (1984) [Sov. Phys. Solid State 26, 1890 (1984 )].

[12] G. A. Alers, Phys. Rev. 119, 1532 (1960).

[13] D. I. Bolef, J. Appl. Phys. 32, 100 (1961).

[14] P. D. Bogdanoff, B. Fultz, and S. Rosenkranz, Phys. Rev. B 60, 3976 (1999).

[15] V. F. Sears, Neutron News 3, 26 (1992).

[16] M. Born and K. Huang, Dynamical Theory of Crystal Lattices (Clarendon Press, Oxford, 1988).

[17] A. Maradudin, E. Montroll, G. Weiss, and I. Ipatova, in Solid State Physics, edited by H. Ehrenreich, F. Seitz, and D. Turnbull (Academic Press, New-York, 1971), Suppl. 3, 2nd ed.

[18] W. H. Press, S. A. Teukolsky, W. T. Vetterling, and B. P. Flannery, Numerical Recipes in $C$ (Cambridge Univ. Press, Cambridge, 1994), 2nd ed.

[19] A. F. Yue et al., Hyperfine Interact. 141/142, 249 (2002).

[20] R. Colella and B.W. Batterman, Phys. Rev. B 1, 3913 (1970).

[21] R. J. Elliott and D.W. Taylor, Proc. R. Soc. London A 296, 161 (1967).

[22] E.T. Teatum, K. A. Gschneider, Jr., and J. T. Waber, Los Alamos National Laboratory Report No. LA-4003, 1968.

[23] P. H. Dederichs and R. Zeller, in Point Defects in Metals II, Springer Tracts in Modern Physics Vol. 87 (SpringerVerlag, Berlin, 1980).

[24] G. A. DeWit and B. N. Brockhouse, J. Appl. Phys. 39, 451 (1968).

[25] A. P. Muller and B. N. Brockhouse, Can. J. Phys. 49, 704 (1971).

[26] D. H. Dutton, B. N. Brockhouse, and A. P. Muller, Can. J. Phys. 50, 2915 (1972).

[27] R. Benz, J. Phys. Chem. Solids 31, 713 (1970). 